\title{
Correction to: ChIP: A Choreographic Integration Process
}

Saverio Giallorenzo, Ivan Lanese, and Daniel Russo

\section{Correction to:}

Chapter "ChIP: A Choreographic Integration Process" in: H. Panetto et al. (Eds.): On the Move to Meaningful Internet Systems, LNCS 11230, https://doi.org/10.1007/978-3-030-02671-4_2

The chapter was inadvertently published without the acknowledgement. This has been added. 